\title{
Clinical Outcomes of Perioperative Geriatric Intervention in the Elderly Undergoing Hip Fracture Surgery
}

\author{
Il-Young Jang ${ }^{1,2}$, Young Soo Lee ${ }^{2}$, Hee-Won Jung ${ }^{3,4}$, Jae-Suk Chang ${ }^{5}$, Jung Jae Kim², Hye-Jin Kim², Eunju Lee \\ ${ }^{1}$ Pyeongchang Health Center \& Country Hospital, Pyeongchang, ${ }^{2}$ Department of Internal Medicine, Asan Medical Center, University of \\ Ulsan College of Medicine, Seoul, ${ }^{3}$ Graduate School of Medical Science and Engineering, Korea Advanced Institute of Science and \\ Technology, Daejeon, ${ }^{4}$ Geriatric Center, Seoul National University Bundang Hospital, Seoul National College of Medicine, Seongnam, \\ ${ }^{5}$ Department of Orthopedic Surgery, Asan Medical Center, University of Ulsan College of Medicine, Seoul, Korea
}

Corresponding Author:

Eunju Lee, $\mathrm{MD}, \mathrm{PhD}$

Division of Geriatric Medicine, Department of Internal Medicine, Asan Medical Center, University of Ulsan College of Medicine, 88 Olympic-ro 43-gil, Songpa-gu, Seoul 05505, Korea

Tel: $+82-2-3010-3308$

Fax: +82-2-476-0824

E-mail: eunjulee@amc.seoul.kr

Received: March 31, 2016

Revised: June 15, 2016

Accepted: June 20, 2016
Background: Conventionally, elderly hip fracture patients are assessed by orthopedists to decide whether they need geriatric intervention. We aimed to evaluate the effect of perioperative geriatric intervention on healthcare outcomes in patients undergoing surgery for hip fractures. Methods: Our care model for hip fracture surgery resembles a combination of a routine geriatric consultation model and a geriatric ward model. We retrospectively reviewed the medical records of patients aged $\geq 65$ years undergoing surgery for hip fracture at a single tertiary hospital from January 2010 to December 2013. We assessed comorbidity, indwelling status, fracture type, and mode of anesthesia. We also evaluated in-hospital expenditure, duration of admission, disposition at discharge and 1-year mortality as clinical outcomes. We developed a propensity score model using the variables of age, cholesterol, and creatinine and examined the effect of perioperative geriatric intervention on intergroup differences of clinical variables. Results: Among 639 patients, 138 patients received the geriatric intervention and 501 patients received the usual care. Univariate analysis showed that factors such as age; Charlson comorbidity index; and serum levels of cholesterol, albumin, and creatinine differed significantly between these 2 groups. There was no significant difference between the groups in terms of 1-year mortality, disposition at discharge, and in-hospital expenditure in the propensity matched model. However, the duration of hospitalization was shorter in the intervention group ( $8.9 \pm 0.8$ days) than in the usual care group ( $14.2 \pm 3.7$ days, $p=0.006)$. Conclusion: This care model of geriatric intervention for patients with hip fracture is associated with reduced hospitalization duration.

Key Words: Hip fractures, Aged, Orthopedic procedures, Geriatric assessment

\section{INTRODUCTION}

Hip fracture is one of the most common reasons for Emergency Department visits in the elderly. With the increasing life span, the number of elderly people undergoing hip fracture surgery has increased, since the rate of hip fracture grows exponentially with age ${ }^{1)}$, with approximately $90 \%$ of hip fractures occurring above 65 years of age ${ }^{2)}$.

There have been several well-designed studies showing a close association between hip fracture and substantial mortality, morbidity, nursing home stays, and socio-economic burden ${ }^{3-8)}$. In spite of considerable medical and technological improvements, hip surgery still results in high morbidity and mortality rates ${ }^{9-11)}$. The importance of a multidisciplinary team approach for improving the outcomes for elderly patients undergoing hip fracture surgery has been emphasized, and standardized management protocols have been proposed. Several care models for the management of hip fracture have been designed, and such team approaches have been shown to be beneficial for the management of some issues ${ }^{12-17)}$. Moreover, the National Institute for Health and Care Excellence clinical guidelines recommend ortho-geriatric assessment at admission as a first step for patients with hip fracture.

In spite of its clinical benefit, organizing an orthopedicgeriatric hip surgery unit is not easy for many institutions, because interdisciplinary team approaches might not only increase medical costs for patients, but also require cooperation between different health care professionals, such as orthopedic surgeons, geriatricians, and allied specialists. For these reasons, the usual clinical practice with older patients with hip fractures is for them to be assessed by the orthopedic 
team to decide whether geriatric intervention is required.

Our hypothesis was that perioperative geriatric intervention, the need for which is currently determined by the orthopedist, would be clinically beneficial compared to the usual care model managed by orthopedists without including geriatricians. We therefore aimed to evaluate the impact of perioperative geriatric intervention at the request of the orthopedist on healthcare outcomes in patients undergoing surgery for hip fracture.

\section{MATERIALS AND METHODS}

\section{Study Design}

This study was a retrospective observational study. We reviewed the medical records of patients over 65 years of age with hip fracture who underwent surgical treatment at a Asan Medical Center from January 2010 to December 2013. Hip fractures were limited to the femur neck and intertrochanteric fractures. Other hip fractures, including distal hip fracture, high-energy hip fracture, pathologic fracture, nontraumatic fracture such as avascular necrosis of the femoral head, and hip fractures with concurrent skeletal fracture, including Colles' fracture, were excluded.

\section{1) Ortho-geriatric care model}

Since 2009, this hospital has a geriatric care center that has employed a multidisciplinary approach to helping in the perioperative management of elderly patients, including consultations and transfers from the emergency room to the admission ward. When a patient visits the Emergency Department with a hip fracture, they are first examined by orthopedic doctors who decide whether geriatric intervention is required or not. All patients aged $\geq 65$ years with $\geq 2$ comorbidities were referred to a geriatric physician. After comprehensive evaluation by a geriatrician, hospital admission and the need for further geriatric intervention were determined at the decision of the geriatrician. Fig. 1 illustrates the medical care process in the Emergency Department.

\section{2) Definition of geriatric intervention}

Comprehensive geriatric assessment was conducted for all patients referred to geriatricians, which served as a multidimensional interdisciplinary diagnostic process to determine the medical, psychological, and functional abilities of elderly people ${ }^{18)}$. Based on the results of the comprehensive geriatric assessment, the geriatrician determined whether the patient would require geriatric intervention. Patients who were determined to require geriatric intervention were admitted to the geriatric or orthopedic ward and received continuous geriatric intervention. Geriatric intervention consists of the follo- wing: initial screening and risk management for physical status, nutritional status, cognitive function, and mood status; prevention of malnutrition, polypharmacy, incontinence, and falls; rehabilitation in the geriatric ward; and discharge planning including a long-term care plan. Other patients in the usual care group were hospitalized in the Department of Orthopedic Surgery. There were no differences between the geriatric intervention group and the usual care group with respect to ordinary perioperative care except for geriatric intervention. Patients admitted to departments other than geriatrics or orthopedic surgery were excluded.

\section{Study Subjects and Baseline Characteristics}

The records of a total of 639 patients aged $\geq 65$ years who underwent surgery for hip fracture were examined. We evaluated baseline characteristics including age, sex, body mass index (BMI), American Society of Anesthesiologists (ASA) physical status classification, Charlson comorbidity index (CCI), and surgical factors including fracture type and mode of anesthesia. The ASA physical status classification is commonly used to subjectively estimate preoperative health status ${ }^{19)}$, and the $\mathrm{CCl}$ evaluates the impact of chronic comorbid medical illnesses on independent functioning; high scores reflect a high number or degree of seriousness of coexisting diseases ${ }^{20)}$. We also recorded hemoglobin level, leukocyte count, serum albumin, cholesterol, and creatinine level.

\section{Outcome Measures}

The primary objective was to assess the clinical benefit of perioperative intervention by geriatricians in elderly hip fracture patients undergoing surgery. Outcomes were com-

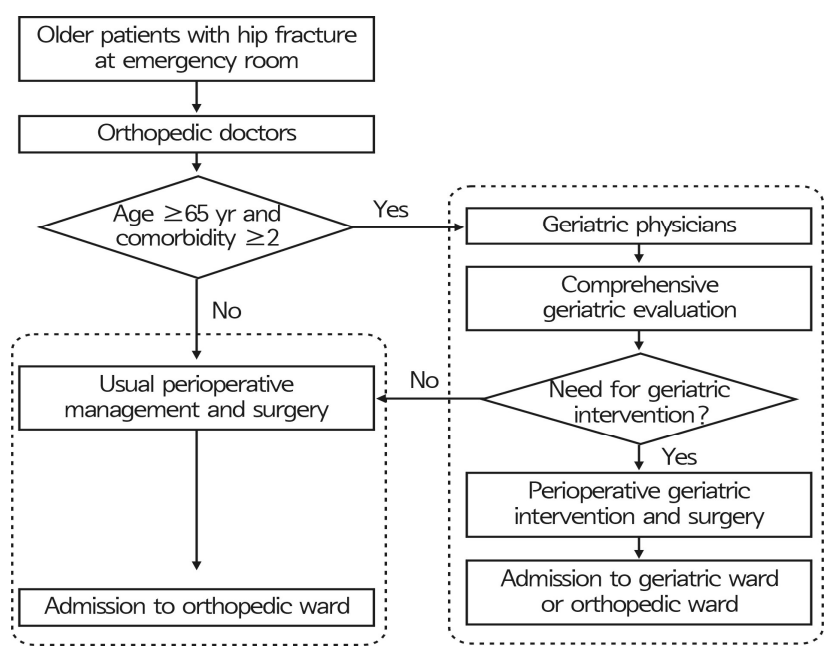

Fig. 1. Initial steps in the management of hip fracture patients in the Emergency Department. The decision regarding whether a geriatrician is needed is completely dependent on the orthope dists' assessment of the patient's medical condition. 
posites of hospital expenditure, duration of admission, patient disposition, and 1-year mortality. We defined 1-year survival as the time from surgery to death within 1 year from any cause. We also evaluated disposition at discharge by identifying the place of residence before and after surgery, such as home, nursing facility, rehabilitation center, general hospital, and death.

\section{Statistical Analysis}

We used propensity score matching to reduce selection bias by matching the subjects in the 2 groups on the basis of their propensity scores, rather than directly comparing individual covariates. We derived the probabilities for receiving geriatric intervention, or propensity scores, using a logistic regression model based on potentially confounding variables: age, sex, fracture site, BMI, ASA physical status classification, $\mathrm{CCl}$, hemoglobin, leukocyte count, serum albumin level, creatinine level, and cholesterol level. First, we performed univariate analyses comparing characteristics between the geriatric intervention group and usual care group (Table 1). We found that age, $\mathrm{CCl}$, and serum levels of cholesterol, albumin, and creatinine were significantly different between the groups. Finally, after propensity score matching with age, cholesterol level, and creatinine level, the intergroup differences were attenuated.

We compared patients' baseline characteristics in the raw sample via t-tests or chi-square tests as appropriate. In the matched cohort, we used paired t-tests, chi-square tests, Kaplan-Meier survival analyses, independent and paired sample log-rank tests, and Cox proportional hazards models to examine hospital expenditure, duration of admission, hospital

Table 1. Univariate analysis of baseline characteristics for the entire patient cohort and the paired propensity matched model

\begin{tabular}{|c|c|c|c|c|c|c|}
\hline \multirow[b]{2}{*}{ Variable } & \multicolumn{3}{|c|}{ Full sample } & \multicolumn{3}{|c|}{ Propensity matched model } \\
\hline & $\begin{array}{c}\text { Usual care group } \\
(\mathrm{n}=501)\end{array}$ & $\begin{array}{c}\text { Intervention } \\
\text { group }(\mathrm{n}=138)\end{array}$ & p-value & $\begin{array}{l}\text { Usual care group } \\
(\mathrm{n}=138)\end{array}$ & $\begin{array}{c}\text { Intervention } \\
\text { group }(\mathrm{n}=138)\end{array}$ & p-value" \\
\hline Age (yr) & 76.9 & 81.7 & 0.000 & 80.9 & 81.7 & 0.316 \\
\hline Sex, women & $367(73.3)$ & $94(68.1)$ & 0.249 & $97(70.2)$ & $94(68.1)$ & 0.697 \\
\hline Body mass index $\left(\mathrm{kg} / \mathrm{m}^{2}\right)$ & 22.2 & 21.7 & 0.116 & 22.1 & 21.6 & 0.791 \\
\hline ASA class & 2.4 & 2.4 & 0.955 & 2.4 & 2.4 & 0.360 \\
\hline CCI score & 2.5 & 3.4 & 0.000 & 3.3 & 3.4 & 0.698 \\
\hline Serum hemoglobin level $(\mathrm{g} / \mathrm{dL})$ & 11.5 & 11.3 & 0.787 & 11.0 & 11.3 & 0.547 \\
\hline Serum WBC count $(\mu \mathrm{L})$ & 9,731 & 9,882 & 0.635 & 9,526 & 9,882 & 0.423 \\
\hline Serum creatinine level (mg/dL) & 1.08 & 1.40 & 0.041 & 1.21 & 1.40 & 0.324 \\
\hline Serum albumin level (g/dL) & 3.4 & 3.3 & 0.002 & 3.3 & 3.3 & 0.489 \\
\hline Serum cholesterol level (mg/dL) & 158.0 & 143.0 & 0.000 & 139.8 & 143.0 & 0.451 \\
\hline No. of femur neck fracture & $234(46.7)$ & $56(40.6)$ & 0.051 & $57(41.3)$ & $56(40.6)$ & 0.342 \\
\hline No. of intertrochanteric fracture & $267(53.3)$ & $82(56.4)$ & - & $81(58.7)$ & $82(59.4)$ & - \\
\hline
\end{tabular}

Values are presented as mean or number (\%).

ASA, American Society of Anesthesiologists physical status classification; CCI, Charlson comorbidity index; WBC, white blood cell. "p-values obtained by t-test and chi-square test.

Table 2. Comparison of clinical outcomes in the geriatric intervention group and the usual care group in the propensity matched model

\begin{tabular}{lccc}
\hline \hline Variable & Usual care group & Intervention group & p-value \\
\hline 1-Year all-cause mortality & $40(29.0)$ & $31(22.5)$ & 0.216 \\
30-Day mortality & $2(1.4)$ & $1(0.7)$ & 0.563 \\
In-hospital mortality & $1(0.7)$ & $0(0)$ & 0.319 \\
Time to surgery (day) & $3.5 \pm 1.8$ & $3.5 \pm 3.2$ & 0.233 \\
Disposition at discharge & $94(68.1)$ & $103(74.6)$ & 0.231 \\
Duration of admission (day) & $14.2 \pm 0.8$ & $8.9 \pm 3.7$ & 0.006 \\
In-hospital expenditure, KRW (USD) & $13,659,683(12,820.0)$ & $11,687,468(10,968.0)$ & 0.189 \\
\hline
\end{tabular}

Values are presented as number (\%) or mean \pm standard deviation unless otherwise indicated.

KRW, Korean Won; USD, United States dollar.

"p-values obtained by t-test and chi-square test. 
discharge and 1-year mortality, respectively. All analyses were carried out using PASW Statistics ver. 18.0 (SPSS Inc., Chicago, IL, USA).

This study was approved by the institutional review board of the Asan Medical Center, Seoul, Korea (approval number: 2013-0944).

\section{RESULTS}

\section{Patient Population}

We identified 639 patients who underwent hip surgery after initial screening; 501 received the usual care and 138 received geriatric intervention. The clinical characteristics of these groups are shown in Table 1. In the univariate analysis, patients in the geriatric intervention group were older, and had a higher mean $\mathrm{CCl}(2.46$ vs. $3.41, \mathrm{p}<0.001)$. These patients also had lower serum albumin ( $3.42 \mathrm{~g} / \mathrm{dL}$ vs. $3.29 \mathrm{~g} / \mathrm{dL}, \mathrm{p}=0.002)$ and serum cholesterol $(158.04 \mathrm{mg} / \mathrm{dL}$ vs. $142.96 \mathrm{mg} / \mathrm{dL}, \mathrm{p}<0.001)$ levels.

The propensity matched groups consisted of 138 patients each, matched on the basis of the available variables, with a resulting c-index of 0.826 . There were no significant differences between their characteristics.

\section{Mortality}

Table 2 shows the results of the matched model. With regard to postoperative all-cause, 1-year mortality, there were 40 deaths in the usual care group and 31 in the geriatric intervention group $(29.0 \%$ vs. $22.5 \%, p=0.216)$. The following factors were significantly associated with mortality in the propensity matched model: age > 85 years (93 patients; $17.8 \%$ in the usual care group vs. $37.5 \%$ in the intervention group, $p=0.034$ ), albumin level $<3.0$

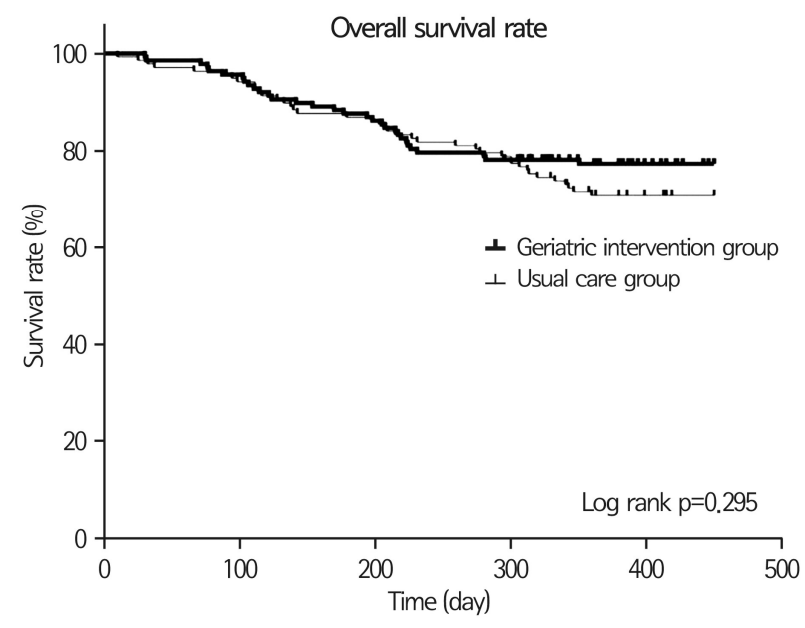

Fig. 2. Kaplan-Meier survival plots (time until death with number of evaluated patients) for the propensity matched geriatric intervention and usual care groups. $\mathrm{g} / \mathrm{dL}$ (65 patients; $50.0 \%$ in the usual care group vs. $24.1 \%$ in intervention group, $\mathrm{p}=0.033)$, and $\mathrm{CCl}$ of $\geq 3$ points ( 150 patients; $39.1 \%$ in the usual care group vs. $21.0 \%$ in the intervention group, $p=0.015$ ). Fig. 2 presents the 1 -year survival curves obtained using the Kaplan-Meier method. Moreover, 30-day mortality and in-hospital mortality were not significantly different.

\section{Morbidities and Other Measurements}

There were no significant differences in discharge disposition (94 cases [68.1\%] vs. 103 cases [74.6\%], p=0.231), hospital expenditure $(13,659,683$ Korean won [KRW] vs. 11,687,468 KRW, $p=$ $0.189)$, and time to surgery $(3.5 \pm 1.8$ days vs. $3.5 \pm 3.2$ days, $p=$ 0.233 ). However, duration of hospitalization was shorter in the intervention group ( $8.9 \pm 0.8$ days vs. $14.2 \pm 3.7$ days, $p=0.006)$.

In a subgroup analysis of duration of hospitalization, age $>75$ years (228 patients; 15.6 days of usual care group vs. 9.5 days of intervention group, $\mathrm{p}=0.034$ ), and $\mathrm{CCl}$ of $\geq 3$ points ( 150 patients; 17.1 days of usual care group vs. 8.8 days of intervention group, $p=0.015$ ) were significantly different in the same model.

\section{DISCUSSION}

The incidence of the hip fracture has been rapidly increasing with the growth of the senior population. Worldwide hip fracture rates are expected to increase to approximately 21 million per year by $2050^{21)}$. Hip fractures usually require surgical treatment, and older people are usually high-risk candidates for major surgery because of their comorbidities and functional deficits. Previous studies have shown that the 1-year mortality rate for hip fracture in the elderly ranges from $20 \%$ to $30 \%{ }^{22,23)}$. Approximately $15 \%$ of the patients die within 6 months and $20 \%$ within 1 year, which is about 3.5 fold higher than the corresponding figures for the general population ${ }^{24)}$. Moreover, the mortality rate increases much more if the patient already has medical comorbidities or suffers postoperative complications. Twenty-five percent of elderly individuals who were independent before fracture remained in nursing homes for more than 1 year, and 60\% required assistance for one or more activities of daily living ${ }^{6,25)}$.

In recent decades, physicians have sought to reverse these poor outcomes of hip surgery. In many studies, functional deficits before hip surgery have been shown to be important risk factors in older patients, as well as geriatric syndromes, which can lead to postoperative delirium ${ }^{26)}$. As residual function declines with physiologic age and deficits accumulate, there is increased need for geriatric medicine, which aims to improve the functioning and well-being of older persons ${ }^{21)}$. Therefore, multidimensional geriatric intervention during the perioperative period aims not only to provide acute medical treatment but also to restore function after the event has occurred $^{27)}$. 
There are several models for geriatric participation in perioperative care of the elderly undergoing hip surgery. Geriatric participation falls into 3 categories: routine geriatric consultation, assignment of the patients to a geriatric ward with orthopedic consultation, and shared care ${ }^{27)}$. According to a recent meta-analysis of the benefits of ortho-geriatric services, geriatric collaboration in general improves outcomes for older patients, and is associated with a significant reduction of in-hospital and long-term mortality ${ }^{26,28)}$. In terms of the individual models, several studies of the geriatric consultation model within orthopedic wards, which is the older and more traditional form, showed a reduced length of stay and much reduced mortality. It is difficult to judge the benefits of the geriatric ward model because of the small number of relevant published reports. The shared model, the later and more advanced model, yields a significant improvement in length of stay.

However, each center analyzed in the meta-analysis has a different health care system, which means each model is slightly different from the others. In addition, the advanced shared care model requires more geriatricians and facilities, which is expected to increase medical costs. To the best of our knowledge, a cost-effectiveness study of the shared care model has not been conducted in Korea. In reality, the number of geriatricians is limited, and geriatric evaluation usually needs a lot of time. We also do not know whether the orthogeriatric shared care unit is economically advantageous. After all, owing to the nature of the hospital-specific health care system and the expected additional medical costs, it is not easy to create a collaborative unit of orthopedists and geriatricians dedicated to elderly patients undergoing hip surgery.

Our transitional care model for hip fracture surgery resembles a combination of the routine geriatric consultation model and the geriatric ward model. The purpose of this study was to evaluate the clinical benefits of geriatric intervention in our new perioperative care model, which begins with the orthopedist's decision to collaborate with geriatricians. To overcome the limitation of a retrospective study, we applied clear standards for hip surgery and geriatric intervention. Although only a limited number of patients were analyzed, there was a significant benefit in terms of length of hospital stay. Hospital expenditure and time to surgery also seemed to improve, but these effects did not reach statistical significance.

Even though we failed to show a significant effect on mortality in our care model of hip fracture, factors like age over 85 years, albumin level less than 3.0, and $\mathrm{CCl}$ of 3 or more were significantly associated with mortality in the propensity matched model. Furthermore, duration of hospitalization, age over 75 years, and $\mathrm{CCl}$ of 3 or more points were significantly different in the same model in a subgroup analysis.
Considering that comorbidity and albumin level are important indicators of frailty in the elderly ${ }^{29)}$, our results support the view that frailty is closely associated with poor outcomes after hip surgery.

Compared with the shared model, our care model for hip surgery has considerable merits. First, it is flexible and applicable to medical centers regardless of their circumstances and departmental structure. It is also beneficial in terms of management costs: fewer human resources and facilities are needed than in the shared model and it does not require any change in the current rigid insurance system. Finally, we found that the orthopedist-oriented decision to collaborate with geriatricians did reduce length of hospital stay.

In conclusion, the findings of this retrospective review show that geriatric intervention for patients with hip fractures is associated with decreased duration of hospitalization compared to usual care. To identify the best model for geriatric collaboration in hip surgery and to clarify the effectiveness of geriatric intervention, future studies should consider the economic aspects and functional benefits of each model including effects on quality of life.

Conflict of Interest Disclosures: The researchers claim no conflicts of interest.

\section{REFERENCES}

1. Scott JC. Osteoporosis and hip fractures. Rheum Dis Clin North Am 1990;16:717-40.

2. Stevens JA, Olson S. Reducing falls and resulting hip fractures among older women. MMWR Recomm Rep 2000;49(RR-2): 3-12.

3. Bentler SE, Liu L, Obrizan M, Cook EA, Wright KB, Geweke JF, et al. The aftermath of hip fracture: discharge placement, functional status change, and mortality. Am J Epidemiol 2009; 170:1290-9.

4. Haentjens P, Magaziner J, Colon-Emeric CS, Vanderschueren D, Milisen K, Velkeniers B, et al. Meta-analysis: excess mortality after hip fracture among older women and men. Ann Intern Med 2010;152:380-90.

5. Johnston AT, Barnsdale L, Smith R, Duncan K, Hutchison JD. Change in long-term mortality associated with fractures of the hip: evidence from the scottish hip fracture audit. J Bone Joint Surg Br 2010;92:989-93.

6. Magaziner J, Hawkes W, Hebel JR, Zimmerman SI, Fox KM, Dolan $\mathrm{M}$, et al. Recovery from hip fracture in eight areas of function. J Gerontol A Biol Sci Med Sci 2000;55:M498-507.

7. Marks R, Allegrante JP, Ronald MacKenzie C, Lane JM. Hip fractures among the elderly: causes, consequences and control. Ageing Res Rev 2003;2:57-93.

8. Wolinsky FD, Fitzgerald JF, Stump TE. The effect of hip fracture on mortality, hospitalization, and functional status: a prospective study. Am J Public Health 1997;87:398-403.

9. Cooper C. Osteoporosis: disease severity and consequent fracture management. Osteoporos Int 2010;21(2 Suppl):S425-9. 
10. Cummings SR, Melton LJ. Epidemiology and outcomes of osteoporotic fractures. Lancet 2002;359:1761-7.

11. Melton LJ 3rd. Adverse outcomes of osteoporotic fractures in the general population. J Bone Miner Res 2003;18:1139-41.

12. Koval KJ, Chen AL, Aharonoff GB, Egol KA, Zuckerman JD. Clinical pathway for hip fractures in the elderly: the Hospital for Joint Diseases experience. Clin Orthop Relat Res 2004; (425):72-81.

13. Choong PF, Langford AK, Dowsey MM, Santamaria NM. Clinical pathway for fractured neck of femur: a prospective, controlled study. Med J Aust 2000;172:423-6.

14. Roberts HC, Pickering RM, Onslow E, Clancy M, Powell J, Roberts A, et al. The effectiveness of implementing a care pathway for femoral neck fracture in older people: a prospective controlled before and after study. Age Ageing 2004;33:178-84.

15. Batsis JA, Phy MP, Melton LJ 3rd, Schleck CD, Larson DR, Huddleston PM, et al. Effects of a hospitalist care model on mortality of elderly patients with hip fractures. J Hosp Med 2007; 2:219-25.

16. Phy MP, Vanness DJ, Melton LJ 3rd, Long KH, Schleck CD, Larson DR, et al. Effects of a hospitalist model on elderly patients with hip fracture. Arch Intern Med 2005;165:796-801.

17. Prestmo A, Hagen G, Sletvold O, Helbostad JL, Thingstad P, Taraldsen K, et al. Comprehensive geriatric care for patients with hip fractures: a prospective, randomised, controlled trial. Lancet 2015;385:1623-33.

18. Rubenstein LZ, Josephson KR, Wieland GD, English PA, Sayre JA, Kane RL. Effectiveness of a geriatric evaluation unit: a randomized clinical trial. N Engl J Med 1984;311:1664-70.

19. Saklad M. Grading of patients for surgical procedures. Anes the- siology 1941;2:281-4.

20. Charlson ME, Pompei P, Ales KL, MacKenzie CR. A new method of classifying prognostic comorbidity in longitudinal studies: development and validation. J Chronic Dis 1987;40:373-83.

21. Gullberg B, Johnell O, Kanis JA. World-wide projections for hip fracture. Osteoporos Int 1997;7:407-13.

22. Leibson CL, Tosteson AN, Gabriel SE, Ransom JE, Melton LJ. Mortality, disability, and nursing home use for persons with and without hip fracture: a population-based study. J Am Geriatr Soc 2002;50:1644-50.

23. Keene GS, Parker MJ, Pryor GA. Mortality and morbidity after hip fractures. BMJ 1993;307:1248-50.

24. Lee YK, Ha YC, Park C, Yoo JJ, Shin CS, Koo KH. Bisphosphonate use and increased incidence of subtrochanteric fracture in South Korea: results from the National Claim Registry. Osteoporos Int 2013;24:707-11.

25. Cooper C. The crippling consequences of fractures and their impact on quality of life. Am J Med 1997;103(2A):12S-17S.

26. Marcantonio ER, Flacker JM, Michaels M, Resnick NM. Delirium is independently associated with poor functional recovery after hip fracture. J Am Geriatr Soc 2000;48:618-24.

27. Grigoryan KV, Javedan H, Rudolph JL. Orthogeriatric care models and outcomes in hip fracture patients: a systematic review and meta-analysis. J Orthop Trauma 2014;28:e49-55.

28. Sohn BK, Sung YB, Park EJ, Lee DW. The incidence and related factors of delirium in elderly patients with hip fracture after surgery. J Korean Geriatr Soc 2010;14:162-70.

29. Kim SW, Han HS, Jung HW, Kim KI, Hwang DW, Kang SB, et al. Multidimensional frailty score for the prediction of postoperative mortality risk. JAMA Surg 2014;149:633-40. 